\title{
Die Psychologie der Schweizer ${ }^{1}$
}

\author{
Thomas Kurz (Zürich)
}

Zusammenfassung: Nach dem Austritt der Jungianer aus der Internationalen Psychoanalytischen Vereinigung (IPV) 1914 hatte Freud in der vormals so wichtigen Provinz Schweiz keine organisierte Gefolgschaft mehr. Erst nach dem 1. Weltkrieg gab es wieder eine Schweizer Ortsgruppe. Bei der Lektüre der Briefwechsel Freuds, der Rundbriefe des "Geheimen Komitees» und dem Studium der Geschichte der Schweizerischen Gesellschaft für Psychoanalyse (SGP) zeigen sich Konstanten, die das Verhältnis der Schweizer zur Freud'schen Internationalen auszeichnen. Immer wieder verweigern sich voll ausgebildete Analytiker dem nationalen (SGP) und internationalen Verband (IPV). Und diejenigen, die mitmachten, offenbarten eine partikularistische Schlagseite, die Freud verbatim als «Kantönligeist» bezeichnete. Sie widersetzten sich Kongresstraditionen, was Kongresse platzen liess, hielten sich nicht an die üblichen Ausbildungsrichtlinien - machten immer alles etwas anders als die Andern. Innerschweizerisch war die Einheit der Freudianer immer wieder vom Partikularismus der Ärzte bedroht. Die Schweizer waren deshalb lange die enfants terribles in der Internationalen Psychoanalytischen Vereinigung. Das letzte Drama in der bald 100-jährigen Geschichte war eine Spaltung, die niemand wollte.

Schlüsselwörter: Schweizerische Gesellschaft für Psychoanalyse, SGP, Internationale Psychoanalytische Vereinigung, IPV, International Psychoanalytical Association, IPA, Geschichte der Psychoanalyse, Rundbriefe, Geheimes Komitee, Spaltung, Lehranalyse, Psychoanalytische Ausbildung.

Als Sigmund Freud im Juli 1914 an Karl Abraham vom Ausbruch des Ersten Weltkriegs schreibt, berichtet er im gleichen Brief vom Ende einer geschlagenen Schlacht: Zürich und die «Schweizer Schule» um Carl Gustav Jung waren gerade aus dem psychoanalytischen Olymp gefallen, oder besser gesagt, hinausmarschiert. Freud hatte erwartet, dass er die Schweizer nur durch die Auflösung der Internationalen Psychoanalytischen Vereinigung (IPV) oder durch kollektiven Austritt und die Gründung einer neuen Organisation loswerden würde. Jung legte das Amt 
des Zentralpräsidenten indessen kampflos nieder und die ruhmreiche Zürcher Ortsgruppe trat aus der IPV aus.

Der Honeymoon mit der Schweiz war zu Ende. Während dem Ersten Weltkrieg hatte Freud in dieser vormals so wichtigen Provinz keine organisierte Gefolgschaft mehr: Der Kontakt beschränkte sich auf Briefverkehr mit dem Zürcher Pfarrer Oskar Pfister und mit dem Psychiater Ludwig Binswanger in Kreuzlingen. Das Verhältnis zur Schweiz war getrübt. 1928 wird er an Binswanger schreiben: «Nein, es steht fest, ich werde die Schweiz nicht mehr sehen» (26.2.1928).

Nach Kriegsende waren Pläne da, Zürich und der Schweiz wieder eine grössere Rolle in der psychoanalytischen Bewegung zuzuerkennen. Es wurde ein Gipfeltreffen in der Schweiz zwischen Hanns Sachs, Otto Rank und Ernest Jones eingefädelt. Jones unterstrich «the immense advantages that Switzerland offers internationally (politically, money, trading, etc.)» (25.3.1919).

Von den Personen, die 1919 die Schweizer IPV-Ortsgruppe, die «Schweizerische Gesellschaft für Psychoanalyse (SGP)», neu gründeten, hielten Freud und seine Entourage allerdings nicht gerade viel. Gegenüber seinem langjährigen «Vize» Sandor Ferenczi beklagte sich Freud, dass Emil Oberholzer Präsident werden solle, «der mir nur als schwerer Neurotiker bedenklich ist. Sie haben in der Schweiz doch eine ganz besondere Reinzucht von Narren» (24.1.1919). Freud wusste, wovon er sprach; Oberholzer hatte bei ihm 1913 eine Zweitanalyse gemacht (Mijolla, 2002). Und Jones berichtete Freud von der Gründungsversammlung: «The best members are Binswanger, a psychiatrist Rohrschach, and Frau Dr. Oberholzer [Emil Oberholzers Frau Mira, Anm, TKJ. Pfister you know; he gives trouble in wanting to make the whole world immediately members, being convinced that every one is on the point of becoming a complete psychoanalyst» (25.3.1919).

AchtWochen nach der Gründung lag die SGP bereits im Clinch mit dem von Freud eingesetzten Mittelsmann, Agent undWanderlehrer (O-Ton Freud) Hanns Sachs und mit der IPV: Sachs hatte den Schweizern geraten, ihr IPV-Beitrittsgesuch wieder zurückzuziehen, da im Verein die Absicht bestünde, «das Moment der Sexualität möglichst unangerührtzu lassen». Einem Brief Freuds an Pfister (27.5.1919) zufolge betrachteten die Schweizer Sachs als «Sendboten der hohen Inquisition». Zu Recht: Freud nahm Sachs mit dem Argument in Schutz, dieser misstraue vielleicht «der «Psychologie der Schweizer und fürchtet, dass die Ver-Jungung bei Ihnen tiefer eingedrungen ist, als Sie alle sich und anderen eingestehen wollen.»

Der Plan, der Schweiz unter Sachs eine besondere Rolle zuzugestehen, wurde bald fallengelassen. Die Schweizer taten in der Folge acht Jahre lang nun alles, um ihrem ramponierten Ruf gerecht zu werden und der IPV inklusive Sigmund Freud 
auf den Nerv zu gehen: Zunächst wurde in Wien, London und Berlin mit Missfallen bemerkt, dass die Romands keine Anstalten zum Beitritt zu SGP und IPV machten. Eduard Claparède hatte 1919 einen offenen psychoanalytischen Kreis gegründet. Jones fragte deshalb in einem Rundbrief im Oktober 1920: "Do you suggest that we make overtures to the other Geneva pseudo-analysts (Claparède, etc), who have refused to join the Swiss group?» Claparède und seine Gruppe blieben draussen.

Ein peinlicher, im nachhinein kaum nachvollziehbarer Konflikt entzündete sich am Zeitschriften-Obligatorium für IPV-Mitglieder. Oberholzer unterbreitete Freud persönlich das Anliegen, dass für die Schweiz eine Ausnahme zu machen sei. Das unsolidarische Ansinnen aus der kriegsverschonten Schweiz kam mithin zu einem Zeitpunkt, als Freud in Wien gegenüber Karl Abraham konstatieren musste, «dass es hier im Zimmer bitterkalt ist» (5.2.1919) und gegenüber Ferenczi, dass der Fleischmangel und chronische Hunger zur Affektmilderung beitrage (25.3.1919). Tatsächlich waren wegen dem Zeitschriftenobligatorium drei Ärzte aus der SGP ausgetreten. Vor allem aber wollte die SGP einen millionenschweren, aber geizigen Schotten mit Palazzo in Genua in ihren Reihen halten. Schliesslich warf Freud den Schweizern vor, dass «sie anstatt die schwierige Situation des Verlages zu bedenken, zu ihren Gunsten eine Begünstigung forderten, die an unsere Existenzmöglichkeit rührte» (an Pfister 25.12.1920).

Im Wirbel um den «ersten psychoanalytischen Roman grossen Stiles», so hatte der Internationale Psychoanalytische Verlag «Der Seelensucher» von Georg Groddeck 1920 angekündigt, waren die Schweizer mit ihrer Kritik zunächst in bester Gesellschaft: Viele kritisierten das Buch, nur Freud fand es «freilich Kaviar fürs Volk, das Werk eines Rabelais ebenbürtigen Kopfes» (an Eitingon, 23.1.1921). Die Schweizer manövrierten sich aber mit sicherem Gespür ins schnelle Abseits, indem sie in Erwägung zogen, das "pornographische Elaborat» selbst anzuzeigen, um die Einfuhr in die Schweiz zu verhindern. In einer zehnseitigen Standpauke schrieben Freud und Otto Rank am 28.2.1921 zu diesem Plan dass sie «ein so ausgiebiges Mass von spiessbürgerlicher Vorsicht und Bravheit wohl nicht vereinbar mit der Würde eines Analytikers gehalten hätten» (Groddeck 1998: 291).

In den zwei folgenden Jahren glänzte dieSGP an Kongressen durch Abwesenheit und im Verein durch Untätigkeit, was Freud bewog, sich bei Pfister über Oberholzer zu beklagen: «Es ist nichts von ihm zu haben. (...) Als Präsident einer Ortsgruppe erscheint er nicht auf dem Kongreß, wahrscheinlich auch nicht auf dem nächsten, und das ist wirklich unerhört» (27.7.1922). 
Von April bis September 1923 fanden überhaupt keine Sitzungen statt (IZP 9, 1923: 551). Im Oktober 1922 schrieb Pfister an Freud endlich, «dass nun in unserer schweiz. Gesellschaft wieder flotte Ordnung herrscht» (23.10.1923).

Ob das besser war? Während die psychoanalytische Welt von der im April 1923 diagnostizierten Krebserkrankung Freuds erschüttert wurde, zügelte Präsident Oberholzer seine Praxis an den Utoquai 39 (Tür an Tür zum späteren Epizentrum der Freud'schen Psychoanalyse in der Schweiz, der Praxis Parin-Morgenthaler am Utoquai 41) und die SGP begann zum ersten, aber nicht zum letzten Mal einen Kongress zu organisieren, der nicht stattfinden sollte: Oberholzer knüpfte die Durchführung der 1926 in Luzern vorgesehenen Tagung zum Erstaunen der IPV an Voraussetzungen, worauf sich die Verhandlungen zerschlugen - der Kongress wurde in Bad Homburg durchgeführt. 1927 versuchte es die IPV nocheinmal mit einem Kongress in der Schweiz, biss aber bei Oberholzer wiederum auf Granit. IPV-Präsident Max Eitingons Fazit: «Mit diesem Representative Man unserer Schweizer ist also nichts zu machen» (Rundbrief, 21.11.1926). Der Kongress 1927 fand in Innsbruck statt.

Oberholzers Meisterstück sollte indessen erst noch kommen: 1926 hatte Freud mit seiner Schrift «Zur Frage der Laienanalyse» versucht, den amerikanischen Bestrebungen, Nichtärzte von der psychoanalytischen Praxistätigkeit auszuschliessen, vor den Bug zu schiessen. Was sich die Amerikaner noch lange nicht getrauten, machte Oberholzer ohne Zögern: Ohne Rücksprache mit Freud oder der IPV trat er 1928 als Präsident zurück, aus der SGP aus und gründete mit Rudolf Brun eine «Schweizerische Ärztegesellschaft für Psychoanalyse» als SGP-Konkurrenz. Oberholzer und Brun sahen einen formalen, lockeren Anschluss an die IPV vor, «um uns in der Wahrung unserer Interessen nach allen Seiten freie Hand zu lassen» (Rundschreiben Oberholzer/Brun, 8.12.1927, zitiert in Schröter, 2004, 578).

Der Partikularismus der Ärzte superponiert auf dem Partikularismus der Schweizer. Freud kannte die Diagnose für diese schweizerische Ur-Krankheit: «Kantönligeist». An Kielholz schrieb Freud, «die Schweizer mit ihrer Spaltung seien doch Partikularisten. Der Kantönligeist spiele da offenbar eine Rolle» (Kielholz 1957: 401 f.). Bereits 1921 war Freud in «Massenpsychologie und Ich-Analyse» auf die «Kantönli» zu sprechen gekommen: «Jedes Kantönli sieht geringschätzig auf das andere herab», schrieb er im Zusammenhang damit, dass "fast jedes intime Gefühlsverhältnis zwischen zwei Personen von längerer Dauer» einen Bodensatz von feindseligen Gefühlen enthalte (Freud 1976: 110 f.).

Das 41-seitige IPV-Aufnahmegesuch Oberholzers wurde von IPV-Präsident Eitingon postwendend abgelehnt. Zur Schweizer Urangst vor Majorisierung und vor 
fremden Richtern meinte Eitingon: «Unterzeichnetem sind aus der Geschichte der I.P.V keine Beispiele von Majorisierungen von Minoritäten bekannt, denen eine neu gegründete Gesellschaft von vornherein vorbeugen zu müssen genötigt sein könnte, ebensowenig ist es ihm ersichtlich, welche Beschlüsse oder Gepflogenheiten der I.P.V z. B. für die Schweiz als «kleineren Staat» unannehmbar seien» (1.3.1928, zitiert in Schröter, 2004, 960).

Zehn Tage zuvor hatte Freud Ernest Jones geschrieben: «We had a good deal of correspondence about the Swiss «Sturm im Wasserglas»» (18.2.1928).

Nach acht Jahren im Vorstand wurde der Basler Arzt Philipp Sarasin neuer Präsident der SGP. Er hatte 1915 bei Bleuler im Burghölzli gearbeitet und die erste Analyse auf der falschen Seite, beim Jungianer Franz Riklin, gemacht, eine zweite bei Hanns Sachs und eine dritte 1921 bei Freud selbst (Mijolla, 2002); 1922 arbeitete er an der neu eröffneten Wiener Poliklinik. Im Juli 1928 besuchte Sarasin Freud in Wien; Freud schrieb danach erleichtert: «Es ist doch so, als ob man die Schweiz wie der gewonnen hätte» (an Eitingon, 18.7.1928). Philippe Sarasin lotste die SGP erfolgreich in ruhigere Gewässer. Dass der im Detail in Interlaken geplante IPVKongress 1931 wiederum ins Wasser fiel, war die Folge der Wirtschaftskrise: Die Schweiz war zu teuer. Er fand schliesslich 1932 in Wiesbaden statt.

Inzwischen war die Schweiz bereits vom Land der Tuberkulosetherapie zum Land des Exils mutiert. Im März 1933 beschwor Ferenczi Freud, mit Patienten und Tochter Anna nach England zu emigrieren, während er selbst beabsichtige, rechtzeitig nach der Schweiz zu reisen. Freud bat Ferenczi zu bedenken, «wie wenig Behaglichkeit das Leben in der Fremde, sei es Schweiz oder England, den Flüchtlin gen verspricht» (2.4.1933). Zwei Wochen nach der Verbrennung seiner Bücher in Berlin schrieb Freud an Pfister: «Zu den gastlichen Ländern gehört die Schweiz nicht» (28.5.1933).

Immerhin war die SGP im August 1934 zum ersten Mal Gastgeber eines tatsächlich stattfindenden IPV-Kongresses. Die IPV hatte sich mit der Arisierung der Psychoanalyse in Deutschland und der Organisation der Emigration zu befassen. Sie optierte unter Ernest Jones, wie Landsmann Chamberlain, mit Appeasement: Weil unter den Mitgliedern eine Unruhe und Besorgnis entstanden war, dass der KommunistWilhelm Reich das Berliner Institut, die Gesellschaft und seine Kollegen durch seine agitatorischen Aktivitäten in Gefahr bringe, brachte es die IPV in Luzern fertig, Reich loszuwerden, ohne Klarheit darüber, ob er nun ausgeschlossen worden oder selbst ausgetreten war. Nach dem Kongress war er jedenfalls draussen.

Die SGP wandte sich nach dem Luzerner Höhenflug wieder den innerschweizerischen Niederungen zu. Im Zusammenhang mit einem neuen IPV-Auf 
nahmegesuch der Oberholzer'schen Ärztegesellschaft wurde die Möglichkeit einer Wiedervereinigung diskutiert und verworfen. Oberholzer emigrierte 1938 in die USA und der Friedrich Glauser-Analytiker Max Müller übernahm die Leitung der Ärztegesellschaft. Zu ihrem Ende erklärte er in seinen Memoiren: «Sie verschwand sang- und klanglos. Schon vor dem Krieg bewarben sich mehrere unserer Mitglieder um die Wiederaufnahme in die alte Gesellschaft, allen voran Brun, der seinerzeit der lauteste Schreier für die Spaltung gewesen war» (Müller 1982: 73). Nach Müller wurde der «Leichnam» erst 1948 offiziell zu Grabe getragen.

1939 war die Schweizer Gruppe im Begriff, wie von Freud gewünscht, die "grosse Bedeutung in der psychoanalytischen Bewegung» wiederzugewinnen, "die sie zu Anfang derselben hatte» (Freud an Oberholzer, 10.5.1921). Weil es im deutschsprachigen Europa keine Ausbildungsmöglichkeit in Freud'scher Psychoanalyse mehr gab, wurde die Schaffung eines Ausbildungsinstituts ins Auge gefasst: «In Aussicht genommen ist Zürich, Lokal Dr. Boss, Zeit evt. an Samstagnachmittagen je 2-3 Stunden» (16.2.1939). Zudem plante die SGP mutig Vortragszyklen, «die dem Publikum zeigen, dass wir noch existieren» (Vorstandssitzung, 16.2.1939). Auch Freud existierte noch; er starb am 23. September 1939, drei Wochen nach dem Überfall auf Polen.

Die Sache so offensiv mit Vortragszyklen zu vertreten, getraute sich die SGP nach Kriegsausbruch dann doch nicht; der Aufbau des Lehrinstituts war indessen auch in den weiteren Vorstandssitzungen ein Traktandum und wurde auf eine Art skizziert, dass darin bereits die Konturen des späteren «Psychoanalytischen Seminars Zürich (PSZ)» aufscheinen: «Wir organisieren auf nächsten Winter Kurse. Wer sie halten will, soll sich beim U.-A. [Unterrichts-Ausschuss] anmelden, der U.-A. wird diese Kurse dann vertreten. Die «Substanz〉 ist das Kurs-Material, d.h. die organisatorischen Fragen entstehen mit den Kursen und können dann, wenn sie aktuell sind, erledigt werden. Die Vorlesenden schauen sich $-z B$. unter ihren ehemaligen Analysanden, nach Hörern um, dann kann eine Hörerliste aufgestellt werden. Die Vorlesenden würden von der Hörerliste in Kenntnis gesetzt, ebenso müssten sie sich vor Beginn ihrer Vorlesungen miteinander über ihre Absichten vereinbaren, damit nicht 〈doppelt genäht werde» (Vorstandssitzung, 24.6.1939).

Die Gründung eines «Schweizerischen Instituts für Psychoanalyse» (Vorstandsprotokoll 14.1.1951) war auch nach Kriegsende weiter auf der SGP-Traktandenliste (neben der Vorbereitung des ersten IPV-Nachkriegskongresses 1949 in Zürich). Wie schwierig ein solches Unterfangen in der Schweiz war, zeigten indessen die niederen Geschäfte, mit denen sich Sarasin herumschlagen musste: Kaum war der Krieg zu Ende, kam es zu erneuten Irritationen zwischen Deutschweizern und 
Welschen: «Es sind Anzeichen vorhanden, dass sich die Welschen von uns separieren wollen, aber alles ist noch unabgeklärt», schrieb Hans Zulliger ins Protokoll vom 19.1.1946. Die Befürchtung bewahrheitete sich nicht.

Dass qualifizierte Personen zögern, ihre Vorträge zu halten und in die SGP einzutreten, war gemäss Vorstandsprotokoll 20 Jahre nach 1919 (und 20 Jahre vor 1968, s.u.) wieder ein Thema: Im Protokoll vom 24.1.1948 bemängelte derVorstand die man gelnde Beitrittswilligkeit von potentiellen Mitgliedern und Disziplinlosigkeit bei den Mitgliedern, «weil einzelne Mitglieder unserer Gesellschaft drauflos Lehranalysen veranstalten, ohne vorherden Unterrichts-Ausschluss zu begrüssen.» Sarasin zählte 1947 36 Lehr- und Kontrollanalysen und musste an derVorstandssitzung vom 24.1.1948 fragen: «Wer sind diese 36 Leute?» Zwei dieser 36 hatten allerdings - nach einer Analyse beim verlorenen Sohn Rudolf Brun - eine IPV-konforme Ausbildung: Paul Parin wurde im Februar 1951, Fritz Morgenthaler ein Jahre später ordentliches Mitglied der SGP.Während Parin ab 1949 volle 16 Jahre als Revisor abverdiente, sass Morgenthaler 1956 im SGP-Vorstand, dafür wurde Parin 1967 Präsident.

Zurück ins Jahr 1951: Der SGP-Vorstand erklärte sich einverstanden, «dass die innerhalb der Gesellschaft bis jetzt bestehenden Einrichtungen zum Studium der Freudschen Psychoanalyse ausgebaut werden im Sinne eines «Schweizerischen Psychoanalytischen Lehrinstitutes .» Pfister hatte die Idee 1949 wieder aufgegriffen und bereits Statuten ausgearbeitet (in welchem er auch sein Anliegen eines Analytiker-Diploms wieder einbrachte). Die Betriebsamkeit hing damit zusammen, dass zwischen verschiedenen tiefenpsychologischen Richtungen ein Rennen im Gang war: «Unter Mitarbeit unseres Mitgliedes Prof. Schneider hat sich ein «schweizerisches Szondi-Komitee` gegründet. Auch das Jung-Institut floriert. Deshalb wird das Einrichten eines «Instituts für Psychoanalyse immer dringender» (19.12.1952).

In den folgenden Jahren verschwand das Traktandum aus den noch vorliegenden Protokollen des Vorstandes. Dafür nahmen Gespräche über Aufnahmegesuche deutlich zu, und damit Diskussionen, welche Analysen von der SGP als Lehranalysen anerkannt würden. Es gab ausserordentliche Mitglieder, die potentielle Analytiker analysierten, obwohl dafür ordentliche Mitglieder vorgesehen waren, und es gab ordentliche Mitglieder, die im Begriff waren, eine eigene Schule zu gründen - die Lehranalysen machten, welche die SGP nicht anerkennen wollte (Gustav Bally und Medard Boss). Sarasin löste das Problem der Dispziplinlosigkeit der Schweizer pragmatisch in zwei Schritten: Zunächst wurde festgehalten, dass der Umstand, dass der Analytiker nicht or dentliches Mitglied der Gesellschaft sei, kein Grund dafür darstelle, den Status der «Lehranalyse» zu verweigern; dass aber «bei ausserordentlichen Mitgliedern die Kontrolle am Kandidaten selbst vorgenommen werde, 
bevor man sie akzeptiere» (29.9.1956). Wegen Boss und Bally wurde dieses Vorgehen auch auf Analysen von ordentlichen Mitgliedern ausgeweitet, was die unten ausgeführte Praxis der nachträglichen Anerkennung von Lehranalysen (nach Fritz Meerwein «eine schweizerische Eigenart», von uns als «Lex Sarasin» bezeichnet) begründete.

Im Januar 1959 beschloss der vollständigeVorstand (Sarasin, de Saussure, Rambert, Blum und Morgenthaler) zusammen mit dem Präsidenten des deutschschweizerischen Unterrichtsausschusses Paul Parin schliesslich einhellig, dass Kandidaten künftig empfohlen werden solle, zwei Besuche bei einem Mitglied des UnterrichtsAusschuss zu machen: Einen Besuch am Anfang der Analyse, um ein Bild von den Symptomen und von dem Wunsch, Psychoanalytiker zu werden, zu bekommen. Und ein zweiter Besuch bei fortgeschrittener Analyse, um den Verlauf beurteilen zu können. Diese Praxis war von de Saussure vorgeschlagen und von Parin portiert worden. Von de Saussure wurde auch übernommen, dass der Analytiker des Kandidaten nicht in den Entscheidungsprozess einbezogen werden solle.

Im Vorstandsprotokoll vom Januar 1959 kam auch zum Ausdruck, dass die Idee eines Instituts für Psychoanalyse - seit Jahren nicht mehr erwähnt - von der Basis aufgegriffen und in Zürich, Bern, Lausanne und Genf in die Tat umgesetzt worden war. Effektiv wurden in Bern seminaristischeVeranstaltungen für Psychiatrieassistenten und in Lausanne solche für Kinderanalytiker gegeben; zudem gab es in Genf ein centre d'enseignements mit wöchentlichenVeranstaltungen. Das «Seminar Zürich» hatte gemäss Bericht von Parin seit November 1958 eigene Räume an der Rämistrasse. «Im Seminar sind ca 15 Hörer, fast alles Nicht-Mediziner», während sich die Ärzte um das Burghölzli (d.h. um das Institut für ärztliche Psychotherapie von Boss und Bally) gruppieren würden. Bereits 1958 zeichnete sich eine Zusammenarbeit zwischen diesen zwei Institutionen ab; der spätere SGP-Präsident Fritz Meerwein war auf der Seite des Boss-Bally-Instituts an den Verhandlungen mit Parin und Morgenthaler beteiligt. 1961 anerkannte die SGP das centre romand d'enseignement psychoanalytique in Genf und das Psychoanalytische Seminar Zürich (PSZ) als offizielle Ausbildungsstätten der SGP. 1961 wurde Raymond de Saussure (auf Vorschlag von Parin) neuer Präsident.

Die Deutschschweizer Psychoanalyse war seit einigen Jahren von Parin (Präsident des Unterrichtsausschusses) und Morgenthaler (Aktuar im Vorstand) geprägt worden; zunächst spannungsfrei und im guten Einvernehmen mit den übrigen Mitgliedern der Gesellschaft. Erst 1962 kam es - von unerwarteter Seite zu einer ersten Missfallenskundgebung über die Machtkonzentration am Utoquai 41 (der Praxis Parin-Morgenthaler): Ausgerechnet der Parin- und Morgenthaler- 
Analytiker Rudolf Brun drohte, aus der SGP auszutreten, weil der Vorstand einem seiner Analysanden die ausserordentliche Mitgliedschaft verweigert hatte. Brun denunzierte den Kreis um Parin und Morgenthaler als «Clan» und empfahl seinen zwei Ex-Analysanden echauffiert eine Nachanalyse bei Zulliger oder de Saussure. Brun konnte schliesslich von seinem Austrittsvorhaben abgebracht werden.

An den Kursen des Zürcher Seminars nahmen 1958-1965 rund 40 Personen teil, zehn Dozenten unterrichteten, unter anderen Bally, Berna, von Blarer, Blum, Lincke, Morgenthaler, Ulrich Moser, Parin und Winter. Einen Bruch zwischen der Zürcher Gruppe und der SGP gab es nicht, im Gegenteil: DieVerbandspolitik der SGP wurde stark von den Zürchern bestimmt, die wichtige Aemter innehatten. Dasselbe trifft für das Verhältnis zur IPV zu: Morgenthaler und Parin engagierten sich im Vereinsleben der IPV, übernahmen Ämter und beteiligten sich an Kongressen.

Das Verhältnis zur IPV war konfliktlos, obwohl die SGP als einzige component society zu Beginn der Ausbildung kein Selektionsverfahren anwandte, was in der IPV bemerkt und von Heinz Kohut moniert worden war. Morgenthaler hatte 1967 aus der Not schweizerischer Disziplinlosigkeit bei der Einhaltung der IPVAusbildungsstandards (s.o.) eine Tugend gemacht (und damit eine Grundlage für das Auseinanderfallen von SGP und PSZ gelegt): Kandidaten machten die psychoanalytische Ausbildung (die persönliche Analyse und der Besuch von Seminarien z. B. am PSZ) nämlich nunmehr offiziell ausserhalb der SGP; und diese behielt sich vor, ungeeignete Personen auch nach langjähriger Analyse bei SGP-Mitgliedern und Seminarbesuchen abzuweisen. Erst mit der Aufnahme als ausserordentliches Mitglied anerkannte die SGP die persönliche Analyse als Lehranalyse und der Analysierte konnte am Vereinsleben teilnehmen.

Als sich die 68er der Freud'schen Psychoanalyse zuwandten, überstieg die Nachfrage nach Analyseplätzen bei SGP-Mitgliedern das Angebot massiv, und die Ausbildungspraxis wurde weiter liberalisiert: Der damalige SGP-Präsident Paul Parin beispielsweise verwies Ausbildungskandidaten offiziell nun auch an ausgebildete Nicht-SGP-Mitglieder mit dem Hinweis auf die erwähnte «Lex Sarasin»: Dass in der Schweiz die Kontrolle in jedem Fall post festum am Kandidaten selbst vorgenommen werde. Im Februar 1974 wurde diese Praxis im offiziellen SGP-Bulletin festgeschrieben: «Wünscht jemand seine Analyse bei einem Analytiker zu machen, der nicht Mitglied der Gesellschaft ist, oder hat er dies bereits unternommen, wird es wie bei jeder Analyse vom Ergebnis abhängen, ob diese Analyse für die Ausbildung zureichend ist."

Das ordentliche SGP-Mitglied Lambelet erklärte im Juni 1974 in Basel: «In unserer Gesellschaft gibt es weder den Begriff des Lehranalytikers noch denjenigen 
der Lehranalyse. Angehende Mitglieder sind deshalb nicht verpflichtet, ihre eigenen Analysen bei ordentlichen Mitgliedern der Gesellschaft zu absolvieren. Zeigt die Analyse das gewünschte Resultat, spielt es keine Rolle, ob sie bei einem ordentlichen oder ausserordentlichen Mitglied der Gesellschaft oder bei einem anderen Analytiker absolviert worden ist» (Protokoll, 22.6.1974).

Parin wurde 1967 SGP-Präsident. Er hatte diese Präsidentschaft in der Meinung übernommen, dass die Jungen eintreten und das im internationalen Vergleich liberale Erbe der Schweizer Gruppe verteidigen würden. Mit dem Eintritt der Jungen hätte sich das Problem natürlich ergeben. Aber wieder einmal traten voll ausgebildete Freudianer nicht in die Organisation der Freud'schen Psychoanalyse ein. Berthold Rothschild, Irene Brogle, Pedro Grosz, Emilio Modena, Judith Valk, Ilka von Zeppelin und andere hatten ein «Kränzli» der 3. Generation gegründet: die linke «Plattform», ein Joint-Venture von 1968 und Freud'scher Psychoanalyse. Anstatt nun durch die weit geöffneten Türen in die SGP einzutreten, die contéstation in sie hineinzutragen und sie umzugestalten, denunzierten sie SGP und IPV als sklerotisch und gerontokratisch und blieben draussen - offensichtlich eine andere «schweizerische Eigenart», neu legitimiert.

Die Etablierung einer Freud'schen Psychoanalyse ausserhalb der SGP wurde weiter vorangetrieben, als Morgenthaler 1970 mit den studentischen Forderungen ernst machen und durchsetzen wollte, dass das PSZ «als erstes Ausbildungsinstitut einer psychoanalytischen Gesellschaft der I.P.A. [=IPV,Anm. T.K.] von den Studenten selbst übernommen und geführt werden» solle: «Ein ordentliches Mitglied wird auch zukünftig der Schweizerischen Gesellschaft für Psychoanalyse gegenüber die Verantwortung für das Seminar Zürich tragen, sofern es möglich ist, das Seminar so zu führen, dass es den Interessen der Gesellschaft nicht zuwiderläuft. Diese Interessen sind ausschliesslich auf die Förderung des Gedankenguts der Psychoanalyse Freuds ausgerichtet.»

Darunter verstanden nun allerdings nicht alle Beteiligten dasselbe, was in den folgenden Jahren zu heftigen Auseinandersetzungen zwischen linken 68ern, weniger politisierten Teilnehmern und etablierten Analytikern führte. Die allgemeine restaurative Demontage der Errungenschaften von 1968 und die drohende Regulierung der Psychotherapie schürten Ängste. Entlassungen von Psychologen im Hause Gilgen führten zu seminarinternen Auseinandersetzungen, weil sowohl die entlassenen Psychologen als auch der entlassende Chef am Seminar studierten. Und wieder einmal platzte 1973 ein Kongress, den die SGP organisierte:Wiederum waren die beteiligten ausländischen Gesellschaften mit der schweizerischen Programmgestaltung unzufrieden, wieder einmal wollten die Schweizer Personen 
auf dem Podium, die nicht SGP-Mitglieder waren (an einem solchen Ansinnen der Schweizer platzte bereits der Luzerner Kongress von 1926), und wieder einmal wurde die nichtkonforme Schweizer Visitenkarte vom Ausland nicht toleriert: Der Kongress wurde abgeblasen (s. Kurz 1993).

Der Ärger der Deutschen und der Österreicher schürte nun vorbestehende Ängste, der Schweizer Sonderzug würde vom Ausland gestoppt und die SGP könnte die Anerkennung der IPV verlieren. 1975 kam es auf Anregung von SGP-Präsident Fritz Meerwein, der eine Spaltung verhindern wollte, zu einer Aussprache mit dem IPV-Präsidenten Serge Lebovici, "to eradicate the general mistrust in our system» (2.10.1974). Danach schlug eine SGP-interne Arbeitsgruppe eine Statutenänderung vor, welche postulierte, dass künftig Ausbildungszentren nur noch anerkannt würden, wenn im Vorstand SGP-Mitglieder die Mehrheit haben. Dies war ein frontaler Angriff auf die Selbstverwaltung des Zürcher Seminars. Das Postulat wurde im April 1977 von der SGP-Generalversammlung gutgeheissen. Einen Monat später teilte die SGP dem PSZ mit, dass der von der SGP gewählte Unterrichtsausschuss der Region Zürich die von den Teilnehmern des Seminars gewählte Seminarleitung ersetze. Als das Seminar diesen Machtwechsel nicht akzeptieren wollte, schloss die SGP die Räume in Witikon - sie hatte als Mieter das Recht dazu. Damit war die Spaltung, die niemand wirklich wollte, vollzogen. Es gab nunmehr zwei Freud'sche Ausbildungsstätten in Zürich.

Hinter dieser Spaltung standen keine unterschiedlichen Auffassungen über die richtige psychoanalytische Lehre, die Metapsychologie , die Neurosenlehre oder die Behandlungstechnik. Die Divergenz bezog sich vielmehr auf die Organisation der psychoanalytischen Gemeinschaft, auf Fragen der Zugänglichkeit und des Elitarismus, der Selektion und der innere Differenzierung. Dies hätte wohl für eine Spaltung nicht gereicht, hätte nicht die Heftigkeit der Auseinandersetzung bei den Beteiligten Narben hinterlassen, die es verunmöglichten, unter demselben Dach (Freud an Abraham, 4.3.1924) an der Sache weiterzuarbeiten.

Die Platzierung der Lehranalyse ausserhalb der SGP, die Autonomisierung des Zürcher Seminars, das von der Anerkennung der SGP abhängig blieb: Diese waghalsige Konstruktion konnte auf die Dauer nicht gut gehen, sicher jedenfalls nicht in den turbulenten, polarisierten Zeiten nach 1968. Es gehört zur Ironie des Schicksals, dass ausgerechnet die kritischen, linken Freudianer die Zugehörigkeit zur Freud'schen Internationale ohne Not preisgegeben haben - wie figura zeigt in gut schweizerischer Tradition.

Dass es nicht, wie nach der Oberholzer-Sezession, zu einer sanftenWiedervereinigung gekommen ist, hängt damit zusammen, dass die SGP beim grossen 
Reinemachen nebenbei das Privileg der ordentlichen Mitglieder, Lehranalysen durchzuführen, ohne landesübliche Übergangsbestimmungen wieder errichtet und mit der Lex Sarasin eine schweizerische Eigenart aufgegeben hat. Viele voll ausgebildete PSZ-Freudianer hatten dadurch nicht mehr einen konformen eigenen Analytiker und die SGP-Mitgliedschaft fiel ausser Betracht. Die Spaltung, die niemand wollte, hat die Kräfte verzettelt und die Stellung der Freud'schen Analyse in der Schweiz geschwächt.

\section{Fussnote}

Die Zitate zur Freud-Korrespondenz stammen aus den publizierten Briefwechseln, diejenigen zur Geschichte der SGP aus dem SGP-Archiv, BlumZulliger-Stiftung, in Bern. Sie erfolgen mit freundlicher Genehmigung des Archivars, Dr. Kaspar Weber.

\section{Literatur und Quellen}

Abraham, H.C. \& Freud, E.L. (Hrsg.) (1980): Sigmund Freud Karl Abraham. Briefe 1907-1926. Frankfurt am Main: S. Fischer.

Fichtner, G. (Hrsg.) (1992): Sigmund Freund Ludwig Binswanger. Briefwechsel 1980-1938. Frankfurt am Main: S. Fischer.

Freud, S.: Gesammelte Werke. Band XIII. Frankfurt am Main: S. Fischer.

Freud, S.: Briefwechsel Sigmund Freud, Max Eitingon. Band I und II, Schröter, M. (Hrsg.) (2004), Tübingen, edition diskord,

Freud, S.: Briefwechsel Sigmund Freud, Sandor Ferenczi. Band II/2. Falzeder E. \& Brabant. E. (Hrsg.) (1996), Wien: Böhlau.

Freud, S.: Briefwechsel Sigmund Freud, Sandor Ferenczi. Band III/ 1. Falzeder E. \& Brabant. E. (Hrsg.) (2003), Wien: Böhlau.

Freud, E.L. \& Meng, H. (Hrsg.) (1963): Sigmund Freud Oskar Pfister, Briefe 1909 1939, Frankfurt a. M.: S. Fischer.

Gidal, T. \& Friedrich, V. (1990): Die Freudianer auf dem 13. Internationalen Psychoanalytischen Kongress 1934 in Luzern, München:Verlag Internationale Psychoanalyse,

Huber, Chr.: Persönliche Mitteilung. Sigmund Freud Privatstiftung, Bibliothek, Archiv, Berggasse 19, Wien

Jones E. (1962): Sigmund Freud, Leben und Werk, Band 1-3, Bern: Huber.

Kielholz, A. (1957): Persönliche Erinnerungen an Freud. Schweizer Archiv für Neurologie und Psychiatrie, Band 79, Heft 2, 401-404 
Kurz, Th. (1993): Aufstieg und Abfall des Psychoanalytischen Seminars Zürich von der Schweizerischen Gesellschaft für Psychoanalyse, Luzifer-Amor, Band 12, 7-54.

Meyer-Palmedo, I. (Hrsg.) (1993): Briefwechsel Sigmund Freud Ernest Jones. 19081939. S. Fischer: Frankfurt a. M.

Müller, M. (1982): Erinnerungen. Erlebte Psychiatriegeschichte 1920-1960, Berlin: Springer.

Paskauskas, R.A. (Ed.) (1993): The Complete Correspondence of Sigmund Freud and Ernest Jones, Cambridge: Harvard University Press.

Schur, M.(1982): Sigmund Freud. Leben und Sterben. Frankfurt am Main: Suhrkamp.

Wittenberger, G. \&Tögel, Ch. (Hrsg.) (1999): Die Rundbriefe des «Geheimen Komitees». Band 1: 1913-1920, Tübingen: edition diskord.

Wittenberger, G. \&Tögel, Ch. (Hrsg.) (2001): Die Rundbriefe des «Geheimen Komitees». Band 2: 1921, Tübingen: edition diskord.

Wittenberger, G. \&Tögel, Ch. (Hrsg.) (2003): Die Rundbriefe des «Geheimen Komitees». Band 3: 1922, Tübingen: edition diskord.

Wittenberger, G. \&Tögel, Ch. (Hrsg.) (2006): Die Rundbriefe des «Geheimen Komitees». Band 4: 1922, Tübingen: edition diskord.

\section{Anmerkungen}

1 Eine gekürzte Version dieses Textes erschien in der Septemberausgabe 2007 der «Schweizer Monatshefte» im Rahmen der Serie «Zürich, Stadt der Seelenkunde». 